Arq. Bras. Med. Vet. Zootec., v.67, n.4, p.1077-1086, 2015

\title{
Desempenho produtivo de bovinos alimentados com cana-de-açúcar com diferentes níveis de concentrado
}

\author{
[Productive performance of cattle fed sugarcane with different concentrate levels] \\ B.M. Salomão ${ }^{1}$, S.C. Valadares Filho ${ }^{2}$, S.D.J. Villela ${ }^{2}$, S.A. Santos ${ }^{3}$, \\ L.F. Costa e Silva ${ }^{1}$, P.P. Rotta ${ }^{1}$ \\ ${ }^{1}$ Universidade Federal de Viçosa - Viçosa, MG \\ ${ }^{2}$ Universidade Federal dos Vales do Jequitinhonha e Mucuri - Diamantina, MG \\ ${ }^{3}$ Universidade Federal da Bahia - Salvador, BA
}

\begin{abstract}
RESUMO
Foram avaliados o consumo, as digestibilidades totais e ruminais e as taxas de digestão $\left(\mathrm{k}_{\mathrm{d}}\right)$ e de passagem $\left(\mathrm{k}_{\mathrm{p}}\right)$ ruminal dos nutrientes de dietas constituídas de cana-de-açúcar in natura e diferentes níveis de concentrado. Utilizaram-se cinco bovinos mestiços, fistulados no rúmen, com peso corporal inicial de $300 \pm 50 \mathrm{~kg}$, distribuídos em delineamento em quadrado latino 5x5. As dietas experimentais foram constituídas de: 1) $100 \%$ cana-de-açúcar in natura (CA); 2) $80 \%$ de CA + 20\% de concentrado (C); 3) $60 \%$ de $\mathrm{CA}+40 \%$ de C; 4) $40 \%$ de $\mathrm{CA}+60 \%$ de $\mathrm{C}$; e 5) $20 \%$ de CA $+80 \%$ de $\mathrm{C}$. Os dados foram analisados utilizando-se o procedimento MIXED do SAS (versão 9.1), bem como análise de regressão e $5 \%$ como nível crítico de probabilidade para o erro tipo I. O consumo de matéria seca (MS), expresso em $\mathrm{kg} / \mathrm{dia}$ ou $\mathrm{g} / \mathrm{kg}$ de peso corporal foi influenciado $(\mathrm{P}<0,05)$ pelos níveis de concentrado. Os demais consumos calculados em $\mathrm{kg} /$ dia também foram influenciados $(\mathrm{P}<0,05)$ pelos níveis de concentrado, assim como os consumos de FDNcp, FDNi e NDT, expressos em $\mathrm{g} / \mathrm{kg}$ de peso corporal. O pool (kg/dia) foi aumentado linearmente à medida que se elevaram os níveis de concentrado para MS. Comportamento similar foi observado para $\mathrm{k}_{\mathrm{i}}\left(\mathrm{h}^{-1}\right)$ da MS e da FDNcp. As taxas de digestão $\left(\mathrm{k}_{\mathrm{d}} \mathrm{h}^{-1}\right)$ da $\mathrm{MS}$, da MO e da FDNcp aumentaram linearmente com a elevação dos níveis de concentrado na dieta. Estimaram-se máximos fluxos omasais (kg/dia) com 52,22 e 31,45\% de concentrado na dieta, respectivamente, para MS e FDNi. A digestibilidade aparente (DA) da MS também foi influenciada pelos níveis de concentrado na dieta. As digestibilidades ruminais da MS e da FDN aumentaram linearmente $(\mathrm{P}<0,05)$ com a adição de níveis crescentes de concentrado. Conclui-se que níveis próximos de $65 \%$ de concentrado resultam em maior ingestão de energia em dietas contendo cana-de-açúcar.
\end{abstract}

Palavras-chave: consumo, taxa de passagem, eficiência microbiana

\begin{abstract}
An experiment was conducted to evaluate the feed intake, total and ruminal dietary component digestibility, ruminal digestion $(k d)$ and passage rates $(k p)$ of cattle fed sugarcane with different levels of concentrate. Five crossbred (Holstein $x$ Zebu) with ruminal cannulas and initial body weight of $300 \pm 50 \mathrm{~kg}$ were used in this experiment. Steers were assigned into a $5 \times 5$ Latin Square design balanced for residual effects. The experimental periods were divided into $16 d$ with $8 d$ for adaptation and $8 d$ for sample collection. Four treatments were used: 1) $100 \%$ fresh sugarcane (SC); 2) $80 \%$ SC + $20 \%$ concentrate (C); 3) $60 \% \mathrm{SC}+40 \% \mathrm{C}$; 4) $40 \% \mathrm{SC}+60 \% \mathrm{C}$; 5) $20 \% \mathrm{SC}+80 \% \mathrm{C}$. Urealammonium sulfate was used at 9:1 ratio to correct the protein level of sugarcane. A regression analysis was performed using the MIXED procedure. Dry matter intake (DMI) expressed either as $\mathrm{kg} / \mathrm{day}$ or $\mathrm{g} / \mathrm{kg}$ of BW was affected $(P<0.05)$ by concentrate level and the maximum intake was estimated to be 63.61 and $75.04 \%$ of concentrate respectively. Intake ( $k g /$ day) of $O M, C P, N D F a p, T D N, i N D F$, digestible NDFap (NDFapD),
\end{abstract}

Recebido em 17 de fevereiro de 2014

Aceito em 27 de março de 2015

E-mail: murtabernardo@ hotmail.com

Projeto de pesquisa financiado pelo CNPq, FAPEMIG e INCT Ciência Animal 
and NFC were affected by concentrate level $(P<0.05)$ and their maximum intake was estimated to be $63.21 ; 63.78 ; 53.14 ; 64.31 ; 32.49 ; 88.47$ e 55.46\% of concentrate respectively. The ruminal pool size of $D M$ and $C P$ as well as ki (h-1) DM, CP, OM, NDFap, and NFC increased linearly as the dietary levels of concentrate increased. The ruminal digestion rate ( $\mathrm{kg} \mathrm{h-1)}$ of DM and NDFap as well as the passage rate $(\mathrm{kg} \mathrm{h}-1)$ of CP increased linearly as the dietary levels of concentrate increased. The maximum omasal flux (kg/day) of DM and iNDF were estimated to be 52.22 and $31.45 \%$ of dietary concentrate respectively. Apparent digestibility of DM, EE, and NFC also increased linearly $(P<0.05)$ with the increase of concentrate levels. Data suggest that levels up to $65 \%$ of concentrate result in greater energy intake by cattle fed sugarcane based diets.

Keywords: feed intake, passage rate, microbial efficiency

\section{INTRODUÇÃO}

A utilização de cana-de-açúcar como alimento volumoso se destaca em potencial para bovinos de corte, num cenário crescente do número de animais confinados (Anualpec, 2012), ou, ainda, na busca de se contornar a escassez de alimento na época seca do ano, quando as pastagens reduzem drasticamente a produção de biomassa.

A cana-de-açúcar tem se destacado na alimentação de bovinos em razão do baixo custo por unidade de MS produzida, da manutenção do valor nutritivo ao longo do período seco do ano, da maior disponibilidade nos períodos de escassez de forragens nas pastagens e do melhor desempenho econômico em comparação a outras forrageiras (Magalhães et al., 2004). Entretanto, quando o objetivo é o alto desempenho dos animais, apresenta algumas limitações, pois a baixa digestibilidade da fibra (Magalhães et al., 2004) ou o alto teor de FDNi podem comprometer o consumo voluntário dos ruminantes, mesmo apresentando teor médio de fibra em detergente neutro menor do que a silagem de milho (Valadares Filho et al., 2002).

Para que dietas à base da cana-de-açúcar apresentem resultados positivos de produtividade animal, essas devem ser suplementadas com concentrados, haja vista que esses apresentam efeitos benéficos sobre o consumo voluntário e a digestibilidade total dos alimentos. No entanto, a modificação da relação volumoso:concentrado, no sentido de aumentar a participação da ração concentrada na dieta, deve ser feita de forma criteriosa, o que exige a condução de estudos nos quais se busquem conhecer, com maior precisão, as interações e os impactos produzidos pelo aumento do nível de concentrado no desempenho desses animais.

Em face do exposto, objetivou-se, com este trabalho, avaliar os consumos e as digestibilidades totais e ruminais dos constituintes das dietas, as taxas de digestão e de passagem ruminal em bovinos alimentados com diferentes níveis de concentrado na dieta, tendo como fonte volumosa a cana-de-açúcar.

\section{MATERIAL E MÉTODOS}

O experimento foi realizado no Confinamento de Animais, e as análises químicas no Laboratório de Nutrição de Ruminantes do Departamento de Zootecnia do Centro de Ciências Agrárias da Universidade Federal de Viçosa, em Viçosa Minas Gerais, entre os meses de agosto e dezembro de 2012.

Foram utilizados cinco bovinos mestiços Holandês-Zebu, machos castrados, com peso corporal (PC) médio inicial de $300 \pm 50 \mathrm{~kg}$ e idade média de 20 meses, distribuídos em delineamento experimental em quadrado latino $5 \times 5$, balanceado para efeitos residuais. O experimento foi constituído de cinco períodos experimentais, com duração de 16 dias cada um, sendo oito destinados à adaptação dos animais às dietas e os outros oito para a realização das coletas.

As cinco dietas experimentais foram formuladas com a cana-de-açúcar in natura (Saccharum officinarum L.) e 0; 20;40; 60 e $80 \%$ de concentrado, na base da MS. A cana-de-açúcar foi triturada diariamente, antes da alimentação, e suplementada com a mistura de ureia + sulfato de amônio (9:1), para elevar os teores de proteína bruta do volumoso para $12 \%$ na base da MS. 
Tabela 1. Composição do volumoso utilizado nos períodos experimentais e dos ingredientes do concentrado

\begin{tabular}{|c|c|c|c|c|c|c|c|c|c|c|c|}
\hline \multirow[b]{2}{*}{ Itens } & \multicolumn{11}{|c|}{ Composição química (\%) } \\
\hline & $\bar{\Sigma}$ & $\stackrel{0}{2}$ & $\stackrel{m}{2}$ & 壳 & 莒。 & 仝 & 污 & 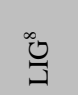 & 言 & $\stackrel{\circ}{\stackrel{4}{1}}$ & 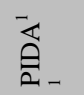 \\
\hline Cana-de-açúcar média & $\begin{array}{c}27,4 \pm \\
1,7\end{array}$ & $\begin{array}{c}96,0 \pm \\
0,7\end{array}$ & $\begin{array}{c}3,0 \pm \\
0,5\end{array}$ & $\begin{array}{c}1,6 \pm \\
0,1\end{array}$ & $\begin{array}{c}51,4 \pm \\
2,5\end{array}$ & $\begin{array}{c}40,3 \pm \\
4,03\end{array}$ & $\begin{array}{c}26,3 \pm \\
0,9\end{array}$ & $\begin{array}{c}4,3 \pm \\
0,2\end{array}$ & $\begin{array}{c}1,59 \pm \\
0,3\end{array}$ & $\begin{array}{c}27,8 \pm \\
1,0\end{array}$ & $\begin{array}{c}0,20 \pm \\
0,14\end{array}$ \\
\hline Fubá de milho & 87,05 & 98,58 & 8,3 & 4,01 & 13,29 & 72,93 & 1,4 & -- & 0,15 & 2,82 & -- \\
\hline Farelo de soja & 88,15 & 93,12 & 52,36 & 2,52 & 16,83 & 21,38 & 0,33 & -- & 1,11 & 6,24 & -- \\
\hline Casca de soja & 88,75 & 94,8 & 13,82 & 1,59 & 58,01 & 21,37 & 3,12 & -- & 5,71 & 39,66 & -- \\
\hline Bicarbonato de sódio & 99,14 & 0,86 & -- & -- & -- & -- & -- & -- & -- & -- & -- \\
\hline Óxido de magnésio & 99,43 & 4,06 & -- & -- & -- & -- & -- & -- & -- & -- & -- \\
\hline Sal mineral & 90,55 & -- & -- & -- & -- & -- & -- & -- & -- & -- & -- \\
\hline Ureia/AS & 98,06 & 99,97 & 261,02 & -- & -- & -- & -- & -- & -- & -- & -- \\
\hline
\end{tabular}

${ }^{1}$ Matéria seca, ${ }^{2}$ matéria orgânica, ${ }^{3}$ proteína bruta, ${ }^{4}$ extrato etéreo, ${ }^{5}$ fibra em detergente neutro corrigida para cinzas e proteína bruta, ${ }^{6}$ carboidratos não fibrosos, ${ }^{7}$ fibra em detergente neutro indigestível, ${ }^{8}$ lignina, ${ }^{9}$ proteína insolúvel em detergente neutro (\%MS), ${ }^{10}$ fibra em detergente ácido, ${ }^{11}$ proteína insolúvel em detergente ácido.

O concentrado utilizado em todas as dietas foi constituído de $47,25 \%$ de fubá de milho; $4 \%$ de farelo de soja; $47,25 \%$ de casca de soja, $1,0 \%$ de bicarbonato de sódio e $0,5 \%$ de óxido de magnésio. A composição do concentrado e das dietas experimentais encontra-se na Tab. 2.

O fornecimento das dietas foi realizado duas vezes ao dia, às oito e às 16 horas, sendo a metade do volumoso e do concentrado em cada horário.

A dieta fornecida foi ajustada para manter as sobras em torno de 5 a $10 \%$, com base na matéria natural, porém estas foram misturadas novamente no dia seguinte à nova dieta, implantando um sistema de alimentação sem retirada de sobras.

A cana picada foi amostrada durante o período de coletas (do oitavo ao $14^{\circ}$ dia) e, posteriormente, identificada, armazenada e congelada a $-20^{\circ} \mathrm{C}$. Todo o material foi submetido à pré-secagem em estufa, com ventilação forçada de ar a $60^{\circ} \mathrm{C}$, durante 72 horas. Após serem secas e moídas, as amostras dos volumosos foram processadas em moinhos de facas tipo Willey, com peneiras de 1 e $2 \mathrm{~mm}$, sendo, em seguida, acondicionadas em potes plásticos e armazenadas para posteriores análises laboratoriais.

Para quantificação dos coeficientes de digestibilidade dos nutrientes, realizou-se amostragem de fezes, realizada no momento da coleta omasal, durante cinco dias consecutivos: do nono ao $13^{\circ}$ dia de cada período experimental.

Posteriormente, as fezes foram colocadas em bandejas de alumínio e pré-secas em estufa, com ventilação forçada de ar, a $60^{\circ} \mathrm{C}$, por 72 horas. Em seguida, foram moídas em moinho de facas tipo Willey, com peneiras de 1 e $2 \mathrm{~mm}$, sendo, então, elaborada uma amostra composta de fezes por animal, em cada período, as quais, posteriormente, foram acondicionadas em potes plásticos e armazenadas para posteriores análises laboratoriais. 
Tabela 2. Proporções dos ingredientes e composição química das dietas experimentais

\begin{tabular}{cccccc}
\hline Itens & \multicolumn{5}{c}{ Níveis de concentrado na dieta $(\%)$} \\
\cline { 2 - 5 } & 0 & 20 & 40 & 60 & 80 \\
\hline${ }^{1}$ Cana-de-açúcar & 100 & 80 & 60 & 40 & 20 \\
Fubá de milho & 0 & 9,45 & 18,9 & 28,35 & 37,8 \\
Casca de soja & 0 & 9,45 & 18,9 & 28,35 & 37,8 \\
Farelo de soja & 0 & 0,8 & 1,6 & 2,4 & 3,2 \\
Bicarbonato de sódio & 0 & 0,2 & 0,4 & 0,6 & 0,8 \\
Óxido de magnésio & 0 & 0,1 & 0,2 & 0,6 & 0,4 \\
\hline & & Composição química das dietas $(\%)^{2}$ & \\
\hline MS & 27,40 & 39,48 & 51,57 & 63,95 & 75,74 \\
MO & 96,00 & 95,83 & 95,65 & 95,49 & 95,30 \\
PB & 12,41 & 12,46 & 12,75 & 12,61 & 12,69 \\
EE & 1,60 & 2,01 & 2,45 & 2,88 & 3,32 \\
FDNcp & 51,40 & 47,27 & 43,92 & 40,56 & 37,20 \\
CNF & 36,45 & 38,79 & 40,35 & 41,79 & 43,27 \\
FDNi & 26,30 & 21,47 & 16,63 & 11,80 & 6,97 \\
PDR & 95,50 & 84,93 & 73,06 & 63,83 & 53,44 \\
\hline
\end{tabular}

${ }^{1}$ Cana-de-açúcar corrigida com ureia/sulfato de amônia ( 96,25\% cana: 3,65\% ureia/sa).

${ }^{2} \mathrm{MS}=$ Matéria seca; $\mathrm{MO}=$ matéria orgânica; $\mathrm{PB}$ = proteína bruta; $\mathrm{EE}=$ extrato etéreo; FDNcp = fibra em detergente neutro corrigida para cinzas e proteína; $\mathrm{CNF}=$ carboidratos não fibrosos; $\mathrm{FDNi}=$ fibra em detergente neutro indigestível; PDR = proteina degradada no rúmen.

Para determinação do fluxo de matéria seca omasal, foram utilizados dois indicadores. O cobalto-EDTA (Co-EDTA) foi usado como indicador da fase líquida e de pequenas partículas e a fibra em detergente neutro indigestível (FDNi) como indicador da fase sólida. O Co-EDTA foi acondicionado em cartuchos de papel e administrado na quantidade total diária de cinco gramas, distribuídos em quatro doses diárias de $1,25 \mathrm{~g}$, nos seguintes horários: às seis, 12,18 e 24 horas. A infusão de Co-EDTA iniciou-se três dias antes da coleta de digesta omasal, que foi efetuada do nono ao $13^{\circ}$ dia de cada período experimental.

As coletas de digesta omasal foram realizadas durante três dias consecutivos, duas vezes ao dia, em intervalos de $12 \mathrm{~h}$ ao dia e de $16 \mathrm{~h}$ entre dias, o que totalizou seis coletas de digesta por período experimental, sendo utilizado um conjunto de dispositivos que consistiu de um kitassato, ou seja, um tubo coletor e uma bomba a vácuo, conforme técnica de Huhtanen (1997), adaptada por Leão (2002).

Ao final de cada período experimental, essas amostras foram descongeladas em temperatura ambiente e filtradas em filtro de náilon a $100 \mu \mathrm{m}$, com área de poros de $44 \%$ de superfície (Sefar Nitex 100/44, Sefar, Thal, Switzerland), obtendo-se, assim, duas fases (sistema de indicador duplo): a fase retida no filtro foi denominada fase de grandes partículas, e a fase filtrada, fase líquida e de pequenas partículas. As amostras das duas fases e uma amostra de digesta sem separação foram identificadas, pesadas em bandeja de alumínio e secas em estufa com ventilação forçada de ar a $60^{\circ} \mathrm{C}$, por 72 horas. Em seguida, as amostras foram moídas em moinho de facas tipo Willey, com peneira de $2 \mathrm{~mm}$, sendo, então, elaborada uma amostra composta de cada fase da digesta, proporcional por animal, em cada período, com base no peso pré-seco total referente a cada coleta. Foi retirada amostra para análise de FDNi, e depois as amostras foram novamente processadas em moinho de facas $(1 \mathrm{~mm})$, sendo, posteriormente, acondicionadas em potes plásticos e armazenadas para posteriores análises laboratoriais.

No $14^{\circ}$ dia de cada período experimental, foi efetuado o esvaziamento total do rúmen ao meiodia, aproximadamente quatro horas após o 
fornecimento da dieta, conforme técnica descrita por Allen e Linton (2007). Após o esvaziamento de todo o conteúdo ruminal, a digesta total foi pesada, posteriormente filtrada em quatro camadas de gaze, para separação das fases sólida e líquida, as quais foram pesadas e amostradas para subsequentes análises químicas. Logo após a amostragem, a digesta ruminal foi novamente reconstituída e colocada no rúmen dos respectivos animais.

No $16^{\circ}$ dia, retornou-se ao mesmo procedimento de esvaziamento, porém, às oito horas da manhã, imediatamente antes do fornecimento da dieta, ou seja, no momento em que o rúmen se encontra, pelo menos teoricamente, em seu menor volume.

As amostras coletadas de digesta ruminal foram pesadas, secas em estufa com ventilação forçada de ar a $60^{\circ} \mathrm{C}$, por 72 horas, moídas em moinho de facas, com peneira de $2 \mathrm{~mm}$, sendo, então, elaborada uma amostra composta por animal em cada período e retirada uma amostra para realizar análises de FDNi. Logo após, o restante das amostras foi novamente moído em moinho de facas, com peneira de $1 \mathrm{~mm}$ para fazer as outras análises. Dessa forma, as amostras compostas foram formadas por amostras da parte sólida e da parte líquida, nos dois esvaziamentos ruminais (antes da alimentação e quatro horas após a alimentação), com base no peso seco de cada amostra, para calcular o pool ruminal de MS e, posteriormente, estimar as taxas de digestão e de passagem dos nutrientes.

As amostras dos volumosos, das sobras, da digesta omasal e do esvaziamento ruminal foram moídas a $2 \mathrm{~mm}$ e submetidas à análise de FDNi em sacos de Ankon $^{\circledR}$ (filter bags F57), por meio de incubação in situ por 288 horas, conforme Detmann et al. (2012).

Todas as amostras dos volumosos, das sobras, das fezes e da digesta omasal foram avaliadas quanto aos teores de matéria seca (MS), matéria mineral (MM), proteína bruta (PB), fibra em detergente neutro corrigida para cinzas e proteína (FDNcp) e extrato etéreo (EE). Somente as amostras de volumosos foram avaliadas quanto aos teores de lignina, e as amostras de esvaziamento ruminal foram avaliadas quanto aos teores de matéria seca (MS) e fibra em detergente neutro corrigida para cinzas e proteína (FDNcp), conforme técnicas descritas por Detmann et al. (2012).

Os dados referentes aos consumos, às digestibilidades totais e parciais, às taxas de digestão e ao pool ruminal de nutrientes foram analisados utilizando-se o procedimento MIXED do SAS (versão 9.1), em que os níveis de concentrado $(0 ; 20 ; 40 ; 60$ e $80 \%)$ representaram o efeito fixo do modelo, enquanto o animal e o período representaram os efeitos aleatórios. As comparações entre os níveis aplicados seguiram a decomposição ortogonal da soma de quadrados associada às fontes de variação em efeitos linear, quadrático, cúbico e quártico e foram conduzidas utilizando-se o PROC MIXED do SAS (versão 9.1). Assumiu-se a homogeneidade das variâncias entre tratamentos, e os graus de liberdade foram estimados pelo método de Kenward-Roger. Os modelos de regressão foram ajustados de acordo com a significância dos parâmetros $\beta 1, \beta 2, \beta 3$ e $\beta 4$, utilizando-se o método da máxima verossimilhança restrita por meio do PROC MIXED do SAS (versão 9.1), e as estimativas deles foram obtidas mediante $\mathrm{o}$ PROC REG do SAS (versão 9.1).

Após avaliação dos efeitos principais, no caso de significância do tempo de coleta, os efeitos linear e quadrático foram avaliados pelo PROC GLM do SAS (versão 9.1), segundo o modelo $\mathrm{Y}=\mathrm{T}+$ $\mathrm{T}^{*} \mathrm{~T}+$ eij, em que $\mathrm{Y}=$ variável resposta, $\mathrm{T}=$ variável contínua relacionada ao tempo de coleta e cij = erro aleatório não observável. Depois de determinado o comportamento da variável resposta, em função do tempo, os respectivos modelos lineares e quadráticos foram obtidos por meio do PROC REG do SAS (versão 9.1). No caso de signficância do efeito do nível de concentrado, as comparações entre os níveis aplicados seguiram a decomposição ortogonal da soma de quadrados associada às fontes de variação em efeitos linear, quadrático, cúbico e quártico e foram conduzidas utilizando-se o PROC MIXED do SAS (versão 9.1). Todos os procedimentos estatísticos foram orientados utilizando-se 0,05 como nível crítico de probabilidade para o erro tipo I. 


\section{RESULTADOS E DISCUSSÃO}

O consumo de matéria seca (MS), expresso em $\mathrm{kg} / \mathrm{dia}$ ou em $\mathrm{g} / \mathrm{kg}$ de peso corporal, apresentou comportamento quadrático $(\mathrm{P}<0,05) \quad \mathrm{em}$ razão do nível de concentrado na dieta (Tab. 3), estimando-se consumos máximos com 63,61 e $75,04 \%$ de concentrado na dieta, respectivamente.

Comportamento quadrático também foi observado $(\mathrm{P}<0,05)$ para o consumo de matéria orgânica (MO), cujo máximo foi estimado com $63,21 \%$ de concentrado na dieta, estando este bem próximo do valor crítico para consumo de MS, expresso em kg/dia. Também houve efeito quadrático $(\mathrm{P}<0,05)$ para os consumos $(\mathrm{kg} / \mathrm{dia})$ de $\mathrm{PB}$, cujo máximo foi estimado com $63,78 \%$ de concentrado (Tab. 3). Já o extrato etéreo (EE) apresentou comportamento linear, significando que quanto maior a participação de concentrado na dieta, maior a quantidade de EE na dieta (Tab. 3).

Tabela 3. Consumo de matéria seca e demais constituintes da dieta de bovinos, alimentados com dietas contendo cana-de-açúcar, com diferentes níveis de concentrado

\begin{tabular}{|c|c|c|c|c|c|c|c|c|c|c|}
\hline \multirow[b]{2}{*}{ Item $^{1}$} & \multicolumn{5}{|c|}{ Concentrado $(\%)^{2}$} & \multirow[b]{2}{*}{ EPM } & \multicolumn{4}{|c|}{ P-valor } \\
\hline & 0 & 20 & 40 & 60 & 80 & & Linear & Quad & $\mathrm{Cub}$ & Quart \\
\hline \multicolumn{11}{|c|}{ Consumo (kg/dia) } \\
\hline $\mathrm{MS}^{3}$ & 3,85 & 5,60 & 7,11 & 8,63 & 8,10 & 0.5174 & $<0.001$ & 0.0016 & 0.0948 & 0.3790 \\
\hline $\mathrm{MO}^{4}$ & 3,57 & 5,23 & 6,71 & 8,15 & 7,65 & 0.4965 & $<0.001$ & 0.0017 & 0.0930 & 0.4139 \\
\hline $\mathrm{PB}^{5}$ & 0,48 & 0,70 & 0,90 & 1,09 & 1,03 & 0.0667 & $<0.001$ & 0.0013 & 0.0915 & 0.4543 \\
\hline $\mathrm{EE}^{6}$ & 0,06 & 0,10 & 0,16 & 0,22 & 0,23 & 0.0118 & $<0.001$ & 0.0966 & 0.0632 & 0.2760 \\
\hline $\mathrm{FDNcp}^{7}$ & 1,84 & 2,52 & 3,09 & 3,39 & 2,97 & 0.2168 & $<0.001$ & 0.0001 & 0.1414 & 0.4852 \\
\hline $\mathrm{NDT}^{8}$ & 2,42 & 3,73 & 4,67 & 5,73 & 4,96 & 0.3212 & $<0.001$ & $<0.001$ & 0.0567 & 0.0667 \\
\hline $\mathrm{FDNi}^{9}$ & 0,94 & 1,11 & 1,14 & 0,99 & 0,56 & 0.0907 & 0.0002 & $<0.001$ & 0.4403 & 0.8740 \\
\hline $\mathrm{FDNd}^{10}$ & 0,90 & 1,47 & 2,02 & 2,68 & 2,74 & 0.1495 & $<0.001$ & 0.0221 & 0.0862 & 0.3094 \\
\hline $\mathrm{CNF}^{11}$ & 1,43 & 2,04 & 2,55 & 2,97 & 2,66 & 0.2092 & $<0.001$ & 0.0007 & 0.1150 & 0.4924 \\
\hline \multicolumn{11}{|c|}{ Consumos $(\mathrm{g} / \mathrm{kg}$ de peso corporal $)$} \\
\hline $\mathrm{MS}^{12}$ & 11,56 & 16,06 & 20,30 & 23,47 & 23,53 & 1.4506 & $<0.001$ & 0.0082 & 0.3391 & 0.8758 \\
\hline $\mathrm{FDNcp}^{13}$ & 5,49 & 7,17 & 8,67 & 9,35 & 8,63 & 6.1808 & $<0.001$ & 0.0032 & 0.3760 & 0.9756 \\
\hline $\mathrm{FDNi}^{14}$ & 2,80 & 3,16 & 3,26 & 2,74 & 1,64 & 0.2403 & $<0.001$ & $<0.001$ & 0.5350 & 0.7586 \\
\hline $\mathrm{NDT}^{7}$ & 7,22 & 11,00 & 13,39 & 15,44 & 14,55 & 0.8683 & $<0.001$ & 0.0002 & 0.4157 & 0.4452 \\
\hline
\end{tabular}

$\mathrm{Y} 3=3.78087+0.15012 \mathrm{X}-0.00118 \mathrm{X} 2\left(\mathrm{R}^{2}=0.8325\right) ; \mathrm{Y} 4=3.50906+0.14413 \mathrm{X}-0.00114 \mathrm{X} 2\left(\mathrm{R}^{2}=0.8351\right) ; \mathrm{Y} 5=$ $0.4711+0.01913 \mathrm{X}-0.00014996 \mathrm{X}^{2}\left(\mathrm{R}^{2}=0.8295\right) ; \mathrm{Y} 6=0.06206+0.00234 \mathrm{X}\left(\mathbf{r}^{2}=0.8694\right) ;$ $\mathrm{Y} 7=2.29403+0.09501 \mathrm{X}-0.00073873 \mathrm{X}^{2}\left(\mathrm{R}^{2}=0,7316\right) ; \mathrm{Y} 8=1.81427+0.06072 \mathrm{X}-0.00057128 \mathrm{X}^{2}\left(\mathrm{R}^{2}=0.7560\right) ;$ $Y 9=0.94532+0.02072 X-0.0003188 X^{2}\left(R^{2}=0.8323\right) ; Y 10=0.8639+0.04395 X-0.00024839 X^{2}\left(R^{2}=0.8802\right) ;$ $\mathrm{Y} 11=1.42277+0.05652 \mathrm{X}-0.00050949 \mathrm{X}^{2}\left(\mathrm{R}^{2}=0.7906\right) ; \mathrm{Y} 12=11.73784+0.31517 \mathrm{X}-0.00210 \mathrm{X}^{2}\left(\mathrm{R}^{2}=0.7454\right) ;$ $\mathrm{Y} 13=0.55743+0.01279 \mathrm{X}-0.00011231 \mathrm{X}^{2}\left(\mathrm{R}^{2}=0.5747\right) ; \mathrm{Y} 14=2.86563+0.0483 \mathrm{X}-0.00072622 \mathrm{X}^{2}$ $\left(\mathrm{R}^{2}=0.7356\right) ; \mathrm{Y} 15=7.32581+0.24296 \mathrm{X}-0.0019 \mathrm{X}^{2}\left(\mathrm{R}^{2}=0.7548\right)$.

${ }^{1} \mathrm{MS}=$ matéria seca; $\mathrm{MO}$ = matéria orgânica; $\mathrm{PB}$ = proteína bruta; $\mathrm{EE}$ = extrato etéreo; $\mathrm{FDNcp}=$ fibra em detergente neutro corrigida para cinzas e proteína; NDT= nutrientes digestíveis totais; FDNpd= FDN potencialmente digestível; $\mathrm{CNF}=$ carboidratos não fibrosos.

${ }^{2}$ Níveis de concentrado na dieta; 0, 20, 40, 60 e 80\% de concentrado na dieta.

O consumo de fibra em detergente neutro corrigida para cinzas e proteína (FDNcp), expresso em $\mathrm{kg} / \mathrm{dia}$ ou $\mathrm{em} \mathrm{g} / \mathrm{kg}$ de peso corporal, apresentou comportamento quadrático $(\mathrm{P}<0,05)$, estimando-se consumos máximos com 53,14 e $56,94 \%$ de concentrado, respectivamente. Comportamento semelhante $(\mathrm{P}<0,05)$ foi observado para o consumo de NDT, expresso em $\mathrm{kg} / \mathrm{dia}$ e $\mathrm{g} / \mathrm{kg}$, estimando-se consumos máximos com 64,31 e $63,94 \%$ de concentrado na dieta (Tab. 3), respectivamente.

$\mathrm{O}$ consumo de fibra em detergente neutro indigestível (FDNi), tanto em $\mathrm{kg} / \mathrm{dia}$ quanto em $\mathrm{g} / \mathrm{kg}$ de peso corporal, também apresentou comportamento quadrático $(\mathrm{P}<0,05)$, estimandose consumos máximos com 32,49 e $33,25 \%$ de concentrado, respectivamente. 
Já o consumo de fibra em detergente neutro digerida (FDNcpD) também apresentou comportamento quadrático $(\mathrm{P}<0,05)$, estimandose consumo máximo com $88,47 \%$ de concentrado na dieta. Comportamento quadrático $(\mathrm{P}<0,05)$ também foi observado para o consumo de carboidratos não fibrosos (CNF), estimandose consumo máximo com $63,93 \%$ de concentrado na dieta (Tab. 3).

Várias pesquisas com cana-de-açúcar como volumoso mostraram efeito negativo no consumo de MS e dos demais nutrientes da dieta. Os pesquisadores apontam o efeito depressivo do consumo relacionado com a baixa digestibilidade da sua fibra (Preston, 1982). Desse modo, uma condição de repleção ruminal ocorrida com animais consumindo esse volumoso pode ser a explicação para redução do consumo (Sampaio $e t$ al., 2009) para os animais alimentados com dieta sem concentrado.

Alguns autores afirmam que fatores de ordem física e fisiológica podem atuar e, assim, controlar o consumo (Van Soest, 1994). Além disso, a qualidade da dieta em termos energéticos e digestivos pode exercer uma resposta tanto positiva quanto negativa (Dias et al., 2000). Esse fato pode explicar a observação de máximo consumo de MS (Tab. 3) à medida que se elevam os níveis de concentrado na dieta, haja vista que houve redução nos teores de fibra indigestível de 24,$42 ; 19,86 ; 16,05 ; 11,05$ e 6,93 para os respectivos níveis de concentrado: $0,20,40,60$ e $80 \%$. Com o incremento de energia digestível na dieta, o consumo tende a aumentar à medida que a taxa e a extensão da digestão aumentam, liberando capacidade no rúmen mais rapidamente. Porém, o consumo é reduzido a partir do momento em que se atinge o limiar energético, quando receptores localizados no rúmen sinalizam para indicar que a exigência energética foi atingida, o que promove redução no consumo de MS, passando da limitação antes física para fisiológica (Forbes, 2003). As estimativas para $\mathrm{k}_{\mathrm{p}} \mathrm{e}$ pool da FDNcp não foram influenciadas $(\mathrm{P}>0,05)$ pelos níveis de concentrado da dieta, entretanto os valores de $\mathrm{k}_{\mathrm{i}}$ e $k_{d}$ apresentaram comportamento linear positivo $(\mathrm{P}<0,05)$ com o aumento nos níveis de concentrado. Houve comportamento quadrático $(\mathrm{P}<0,05)$ para o fluxo omasal da FDNcp, estimando-se fluxo máximo com $40,41 \%$ de concentrado na dieta (Tab. 4).
As estimativas para $k_{p}$ e pool da FDNcp não foram influenciadas $(\mathrm{P}>0,05)$ pelos níveis de concentrado da dieta, entretanto os valores de $\mathrm{k}_{\mathrm{i}}$ e $k_{d}$ apresentaram comportamento linear positivo $(\mathrm{P}<0,05)$ com o aumento nos níveis de concentrado. Houve comportamento quadrático $(\mathrm{P}<0,05)$ para o fluxo omasal da FDNcp, estimando-se fluxo máximo com $40,41 \%$ de concentrado na dieta (Tab. 4).

Não houve influência dos níveis de concentrado $(\mathrm{P}>0,05)$ sobre pool, $\mathrm{k}_{\mathrm{i}}$ e $\mathrm{k}_{\mathrm{p}}$ da FDNi, porém foi observado um efeito quadrático $(\mathrm{P}<0,05)$ no fluxo omasal, estimando-se fluxo máximo com $62,92 \%$ de concentrado na dieta. Esse valor de máximo fluxo de FDNi está de acordo com a máxima ingestão de MS que ocorreu com, aproximadamente, $63 \%$ de concentrado.

O aumento do nível de concentrado na dieta pode determinar mudanças na quantidade de alimento ingerido (Signoretti et al., 1999). Desse modo, o efeito quadrático $(\mathrm{P}<0,05)$ para consumo de MS e FDNcp (Tab. 3) parece ser consequência do atendimento dos requerimentos de energia, e o efeito linear $(\mathrm{P}<0,05)$ para taxa de ingestão (Tab. 4) pode ser explicado pelo aumento da concentração de nutrientes digestíveis na dieta.

Diferentemente do efeito linear $(\mathrm{P}<0,05)$ para $\mathrm{K}_{\mathrm{i}}$ e $K_{d}$ de algumas variáveis presentes na Tab. 4, o fluxo omasal apresentou comportamento quadrático $(\mathrm{P}<0,05)$ para MS, FDNcp e FDNi (Tab. 4). Segundo Fernandes et al. (2003), o fluxo de digesta pelo trato gastrintestinal é determinado pela taxa de passagem ruminal que, no caso de forrageiras tropicais, apresenta baixos valores em razão do alto teor de fibra indigestível. A cana-de-açúcar é reconhecida por apresentar alto teor de $\mathrm{FDNi}$, o que explica o menor valor para fluxo omasal. Assim, o fluxo omasal teve o mesmo comportamento do consumo (Tab. 3), uma vez que a digestibilidade ruminal apresentou aumento linear $(\mathrm{P}<0,05)$ para FDNcp (Tab. 5). O mesmo resultado pode-se observar para o fluxo omasal de $\mathrm{FDNi}$, que também apresentou comportamento quadrático $(\mathrm{P}<0,05)$ (Tab. 4), com um ponto de máximo consumo com $32,49 \%$ de concentrado e o ponto de máxima para o fluxo, com $31,45 \%$ de concentrado (Tab. 4). 
Tabela 4. Parâmetros ruminais da matéria seca, da proteína bruta, da matéria orgânica, da fibra em detergente neutro corrigida para cinzas e proteína, da fibra em detergente neutro indigestível e dos carboidratos não fibrosos de bovinos alimentados com dietas contendo cana-de-açúcar com diferentes níveis de concentrado

\begin{tabular}{|c|c|c|c|c|c|c|c|c|c|c|}
\hline \multirow[b]{2}{*}{ Parâmetros } & \multicolumn{5}{|c|}{ Concentrado $(\%)^{7}$} & \multirow[b]{2}{*}{ EPM } & \multicolumn{4}{|c|}{ P-valor } \\
\hline & 0 & 20 & 40 & 60 & 80 & & Linear & Quad & $\mathrm{Cub}$ & Quart \\
\hline & \multicolumn{5}{|c|}{ Matéria seca } & & & & & \\
\hline $\begin{array}{l}\text { Pool } \\
\text { (kg/dia) }\end{array}$ & 3.609 & 3.872 & 4.332 & 4.372 & 4.719 & 0,5968 & 0.0234 & 0.8311 & 0.9269 & 0.6434 \\
\hline $\mathrm{k}_{\mathrm{i}}^{3}\left(\mathrm{~h}^{-1}\right)$ & 0.051 & 0.064 & 0.070 & 0.082 & 0.075 & 0,0077 & 0.0088 & 0.1932 & 0.5946 & 0.5213 \\
\hline $\mathrm{k}_{\mathrm{p}}^{4}\left(\mathrm{~h}^{-1}\right)$ & 0.031 & 0.036 & 0.040 & 0.038 & 0.033 & 0,0044 & 0.7703 & 0.0987 & 0.9110 & 0.7810 \\
\hline $\mathrm{k}_{\mathrm{d}}^{5}\left(\mathrm{~h}^{-1}\right)$ & 0.020 & 0.028 & 0.030 & 0.045 & 0.043 & 0,0043 & $<0.001$ & 0.4970 & 0.2614 & 0.0762 \\
\hline $\begin{array}{l}\text { Fluxo } \\
(\mathrm{kg} / \mathrm{dia})^{6}\end{array}$ & 2.482 & 3.252 & 4.188 & 3.997 & 3.605 & 0,4633 & 0.0034 & 0.0038 & 0.6761 & 0.3336 \\
\hline $\begin{array}{l}\text { Pool } \\
\text { (kg/dia) }\end{array}$ & 2.320 & 2.487 & $\begin{array}{l}\mathrm{FDNch} \\
2.668\end{array}$ & 2.586 & 2.674 & 0,3731 & 0.2510 & 0.6033 & 0.8284 & 0.7027 \\
\hline $\mathrm{k}_{\mathrm{i}}^{3}\left(\mathrm{~h}^{-1}\right)$ & 0.058 & 0.065 & 0.068 & 0.070 & 0.073 & 0,0036 & 0.0047 & 0.6085 & 0.6371 & 0.9247 \\
\hline $\mathrm{k}_{\mathrm{p}}^{4}\left(\mathrm{~h}^{-1}\right)$ & 0.025 & 0.025 & 0.028 & 0.026 & 0.017 & 0,0033 & 0.2314 & 0.1688 & 0.5427 & 0.8784 \\
\hline $\mathrm{k}_{\mathrm{d}}^{5}\left(\mathrm{~h}^{-1}\right)$ & 0.034 & 0.039 & 0.041 & 0.044 & 0.056 & 0,0041 & 0.0014 & 0.3792 & 0.3062 & 0.9148 \\
\hline $\begin{array}{l}\text { Fluxo } \\
\text { (kg/dia) }^{6}\end{array}$ & 1.215 & 1.453 & 1.639 & 1.729 & 1.130 & 0,1748 & 0.7838 & 0.0022 & 0.1687 & 0.6169 \\
\hline $\begin{array}{l}\text { Pool } \\
\text { (kg/dia) }\end{array}$ & 1.827 & 1.826 & $\begin{array}{c}\text { FDNi } \\
2.014\end{array}$ & 1.6301 & 1.4659 & 0,2951 & 0.1477 & 0.2220 & 0.9629 & 0.3513 \\
\hline $\mathrm{k}_{\mathrm{i}}^{3}\left(\mathrm{~h}^{-1}\right)$ & 0.025 & 0.027 & 0.025 & 0.0250 & 0.0168 & 0,0032 & 0.0819 & 0.1238 & 0.7330 & 0.4477 \\
\hline $\mathrm{k}_{\mathrm{p}}^{4}\left(\mathrm{~h}^{-1}\right)$ & 0.025 & 0.027 & 0.025 & 0.0253 & 0.0169 & 0,0032 & 0.0882 & 0.1153 & 0.6991 & 0.4217 \\
\hline Fluxo & 0.948 & 1.125 & 1.152 & 1.0007 & 0.5731 & 0,0983 & 0.0005 & $<.0001$ & 0.5469 & 0.8935 \\
\hline
\end{tabular}

Pool matéria seca $=3,63868+0.01492 \mathrm{X}\left(\mathrm{r}^{2}=0.1185\right) ; \mathrm{K}_{\mathrm{i}}$ matéria seca $=0,05521+0.00031799 \mathrm{X}\left(\mathrm{r}^{2}=0,2525\right) ; \mathrm{K}$ matéria seca $=0.02040+0.00030043 \mathrm{X}\left(\mathrm{r}^{2}=0.4915\right)$; fluxo matéria seca $=2.38809+0.06770 \mathrm{X}-0.00064827 \mathrm{X}^{2}\left(\mathrm{R}^{2}\right.$ $=0.3173) ; K_{i}$ FDNcp $=0.05992+0.00017374\left(r^{2}=0.3301\right) ; K_{d}$ FDNcp $=0.03332+0.00024481 X \quad\left(r^{2}=0.4167\right)$; fluxo FDNcp $=1.16695+0.02475 \mathrm{X}-0.00030620 \mathrm{X}^{2}\left(\mathrm{R}^{2}=0.2776\right) ; \quad \mathrm{K}_{\mathrm{i}} \mathrm{FDNi}=0.08467-0.00024459 \mathrm{X}+$ $0.00001083 \mathrm{X}^{2}\left(\mathrm{R}^{2}=0.6709\right)$; fluxo $\mathrm{FDNi}=0.93395+0.01578 \mathrm{X}-0.00025080 \mathrm{X}^{2}\left(\mathrm{R}^{2}=0.5675\right)$.

${ }^{1}$ Fibra em detergente neutro corrigida para cinzas e proteína; ${ }^{2}$ fibra em detergente neutro indigestível; ${ }^{3}$ taxa de ingestão; ${ }^{4}$ taxa de passagem; ${ }^{5}$ taxa de digestão; ${ }^{6}$ fluxo omasal; ${ }^{7}$ níveis de concentrado na dieta; $0,20,40,60$ e $80 \%$ de concentrado na dieta.

A digestibilidade aparente (DA) da MS apresentou comportamento quadrático $(\mathrm{P}<0,05)$, com nível crítico estimado com $76,70 \%$ de concentrado $(\mathrm{Y}=0,61892+0,00241 \mathrm{X}-$ $\left.0,00001571 X^{2} ; R^{2}=0,523\right)$, porém, para a digestibilidade ruminal (DR), foi observado um efeito quártico $(\mathrm{P}<0,05)($ Tab. 5).

Rotta (2012), ao trabalhar com silagem de milho e cana-de-açúcar in natura e dois níveis de concentrado, 40 e $60 \%$, observou valores de digestibilidade da MS na ordem de 73,5 e $78,13 \%$ para as dietas com 40 e $60 \%$ de concentrado contendo cana-de-açúcar como volumoso, sendo estes superiores aos valores de digestibilidade encontrados nos respectivos níveis de concentrado deste trabalho (Tab. 5), o que pode ser explicado pela possível melhor qualidade do concentrado utilizado por Rotta
(2012), composto apenas por fubá de milho e farelo de soja. Rotta (2012) observou, ainda, valores de digestibilidade das dietas que tinham a silagem de milho como fonte volumosa de 69,75 e $75,33 \%$ para dietas com 40 e $60 \%$ de concentrado, respectivamente, estando estes abaixo dos valores para digestibilidade das dietas com cana-de-açúcar, o que pode ser uma explicação a observação de que dietas com canade-açúcar possuem maiores quantidades de ureia e carboidratos não fibrosos e uma melhor sincronia da hidrólise da ureia com a degradação dos carboidratos não fibrosos (Xin et al., 2010).

Em relação à FDNcp, foi observado comportamento linear positivo $(\mathrm{P}>0,05)$ para $\mathrm{DA}$ e DR (Tab. 5). Esse comportamento linear positivo para a DA da FDNcp pode ser explicado em razão de o aumento dos níveis de 
concentrado ter resultado na substituição da fibra da cana-de-açúcar de menor qualidade pela fibra da casca de soja. A mesma explicação pode ser considerada para o aumento na DR da FDNcp

Efeito linear na digestibilidade da FDNcp, em razão de níveis crescentes de concentrado na dieta, foi observado por Da Silva (2012), ao realizar um experimento com bovinos alimentados com feno de tifton 85 e níveis crescentes de concentrado na dieta $(17,34,51 \mathrm{e}$ $68 \%$ ), mostrando que o aumento de concentrado na dieta aumentou a digestibilidade da FDNcp da dieta.

Tabela 5. Digestibilidade aparente total e ruminal da matéria seca e dos constituintes de bovinos alimentados com dietas contendo cana-de-açúcar, com diferentes níveis de concentrado

\begin{tabular}{|c|c|c|c|c|c|c|c|c|c|c|}
\hline \multirow[b]{2}{*}{ Item $^{1}$} & \multicolumn{5}{|c|}{ Concentrado $(\%)^{2}$} & \multirow[b]{2}{*}{ EPM } & \multicolumn{4}{|c|}{ P-valor } \\
\hline & 0 & 20 & 40 & 60 & 80 & & Linear & Quad & $\mathrm{Cub}$ & Quart \\
\hline & \multicolumn{10}{|c|}{ Digestibilidade aparente (DA) } \\
\hline MS & 0.62 & 0.67 & 0.68 & 0.72 & 0.71 & 0,0160 & $<.0001$ & 0.0293 & 0.4872 & 0.0813 \\
\hline MO & 0.64 & 0.68 & 0.67 & 0.74 & 0.69 & 0,0193 & 0.0069 & 0.0688 & 0.3025 & 0.0372 \\
\hline FDNcp & 0.48 & 0.53 & 0.55 & 0.59 & 0.62 & 0,0216 & $<.0001$ & 0.5563 & 0.9580 & 0.4639 \\
\hline \multirow[t]{2}{*}{$\mathrm{CNF}$} & 0.85 & 0.87 & 0.86 & 0.86 & 0.77 & 0,0218 & 0.0233 & 0.0114 & 0.3939 & 0.4003 \\
\hline & \multicolumn{10}{|c|}{ Digestibilidade ruminal (DR) } \\
\hline MS & 0.36 & 0.43 & 0.42 & 0.55 & 0.57 & 0,036 & $<.0001$ & 0.6869 & 0.6114 & 0.0345 \\
\hline MO & 0.47 & 0.50 & 0.52 & 0.62 & 0.65 & 0,0214 & $<0.001$ & 0.1790 & 0.2788 & 0.1448 \\
\hline FDNcp & 0.34 & 0.43 & 0.46 & 0.51 & 0.62 & 0,0336 & $<.0001$ & 0.5181 & 0.2481 & 0.9753 \\
\hline \multicolumn{11}{|c|}{$\begin{array}{l}\text { CDMS }=0,61892+0,00241 \mathrm{X}-0,00001571 \mathrm{X}^{2}\left(\mathrm{R}^{2}=0,523\right) ; \mathrm{CDMO}=0.63630+0.00991 \mathrm{X}-0.00059826 \mathrm{X}^{2}+ \\
0.00001267 \mathrm{X}^{3}-8.29635 \mathrm{X}^{4}\left(\mathrm{R}^{2}=0.4108\right) ; \mathrm{CDFDNcp}=0.48831+0.00166 \mathrm{X}\left(\mathrm{r}^{2}=0.5626\right) ; \mathrm{CDCNF}=0.84302+ \\
0.00199 \mathrm{X}-0.00003441 \mathrm{X} 2\left(\mathrm{R}^{2}=0.3290\right) ; \mathrm{CDRMS}=0.36205+0.01504 \mathrm{X}-0.00093182 \mathrm{X} 2+0.00001991 \mathrm{X}^{3}- \\
1.27746 \mathrm{E}-7 \mathrm{X}^{4}\left(\mathrm{R}^{2}=0.5768\right) ; \mathrm{CDRMO}=0.45594+0.00240 \mathrm{X}\left(\mathrm{r}^{2}=0.6970\right) ; \mathrm{CDRFDNcp}=0.48831+0.00166 \mathrm{X}\left(\mathrm{r}^{2}\right. \\
=0.5626) . \\
{ }^{1} \mathrm{MS}=\text { matéria seca; } \mathrm{MO}=\text { matéria orgânica; FDNcp }=\text { fibra em detergente neutro corrigida para cinza e proteína; } \\
{ }^{2} \text { níveis de concentrado na dieta }-0=0 \% \text { de concentrado; } 20=20 \% \text { de concentrado; } 40=40 \% \text { de concentrado; } 60= \\
60 \% \text { de concentrado; } 80=80 \% \text { de concentrado. }\end{array}$} \\
\hline
\end{tabular}

\section{CONCLUSÃO}

Recomenda-se utilizar, aproximadamente, $65 \%$ de concentrado em dietas com cana-de-açúcar para bovinos mestiços, valor suficiente para proporcionar maior ingestão de energia.

\section{REFERÊNCIAS}

ALLEN, M. S.; LINTON J.A.V. In vivo methods to measure digestibility and digestion kinetics of feed fractions in the rumen. In. SIMPÓSIO INTENACIONAL AVANÇOS EM TÉCNICAS DE PESQUISA EM NUTRIÇÃO DE RUMINANTES, 1., 2007, São Paulo. Anais... São Paulo: USP, p. 72-89, 2007.

ANUÁRIO DA PECUÁRIA BRASILEIRA ANUALPEC 2012. São Paulo: FNP, 2012.
DETMANN, E.; SOUZA, M. A.; VALADARES FILHO, S. C. et al. Métodos para análise de alimentos. Visconde do Rio Branco: Suprema, 2012. 214p.

DIAS, H. L. C.; VALADARES FILHO, S. C.; SILVA, J. F. C. et al. Consumo e digestões totais e parciais em novilhos $\mathrm{f} 1$ limousin $\mathrm{x}$ nelore alimentados com dietas contendo cinco níveis de concentrado. Rev. Bras. Zootec., v.29, p.545-554, 2000 .

FERNANDES, A. M.; QUEIROZ, A.C.; PEREIRA, J.C. et al. Fracionamento e cinética da degradação in vitro dos carboidratos constituintes da cana-de-açúcar com diferentes ciclos de produção em três idades de corte. Rev. Bras. Zootec., v.32, p.1778-1785, 2003. 
FORBES, J. M. The multifactorial nature of food intake control. J. Anim. Sci., v.81, supl., p.139144, 2003.

LEÃO, M.I. Metodologias de coletas de digestas omasal e abomasal em novilhos submetidos a três níveis de ingestão: consumo, digestibilidade $e$ produção microbiana. 2002. 57f.. Tese (Doutorado) - Escola de Veterinária, Universidade Federal de Minas Gerais, Belo Horizonte, MG.

MAGALHÃES, A.L.R.; CAMPOS, J.M.S; VALADARESFILHO, S.C. et al. Cana-deaçúcar em substituição à silagem de milho em dietas para vacas em lactação: desempenho e viabilidade econômica. Rev. Bras. Zootec.,v.33, p.1292-1302, 2004.

PRESTON, T.R. Nutritional limitations associated with the feeding of tropical forages. $J$. Anim. Sci., v.54, p.877-884, 1982.

ROTTA, P.P. Desempenho produtivo, exigências nutricionais e avaliação de métodos para estimação de fluxo de digesta em bovinos alimentados com silagem de milho ou cana-deaçúcar. 2012. 173f. Dissertação (Mestrado em Zootecnia) - Universidade Federal de Viçosa, Viçosa, MG.

SAMPAIO, C.B.; DETMANN, E.; LAZZARINI, I.; et al. Rúmen dynamics of neutral detergent fiber en cattle fed low-quality tropical forage and supplemented with nitrogenous compounds. Rev. Bras. Zootec., v.38, p.560-569, 2009.
SIGNORETTI, R.D.; COELHO DA SILVA, J.F.; VALADARES FILHO, S.C. et al. Consumo e digestibilidade em bezerros da raça Holandesa alimentados com dietas contendo diferentes níveis de volumoso. Rev. Bras. Zootec., v.28, p.169-177, 1999.

SILVA, G. S. Desempenho de carcaça de novilhos leiteiros submetidos a dietas com diferentes níveis de concentrado. 2012. 60f. Dissertação (Mestrado em Zootecnia) Universidade Federal Rural de Pernambuco, Recife, PE.

VALADARES FILHO, S.C.; ROCHA JUNIOR, V.R.; CAPPELLE, E.R. Tabelas brasileiras de composição de alimentos para bovinos. CQBAL 2.0. Viçosa, MG: UFV, 2002. 297p.

VAN SOEST, P.J. Nutritional ecology of the ruminant. 2.ed. London: Comstock Publishing Associates, 1994. 476p.

XIN, H.S.; SCHAEFER, D.M.; LIU, Q.P. et al. Effects of polyurethane coated urea supplement on in vitro ruminal fermentation, ammonia release dynamics and lactating performance of Holstein Dairy cows fed a steam-flacked cornbased diet. Asian-Australasian J. Anim. Sci., v.23, p.491-500, 2010. 